TRANSACTIONS OF THE

AMERICAN MATHEMATICAL SOCIETY

Volume 361, Number 3, March 2009, Pages 1189-1208

S 0002-9947(08)04735-1

Article electronically published on October 7, 2008

\title{
SPIKE SOLUTIONS IN COUPLED NONLINEAR SCHRÖDINGER EQUATIONS WITH ATTRACTIVE INTERACTION
}

\author{
E. N. DANCER AND JUNCHENG WEI
}

Abstract. We consider the following elliptic system:

$$
\left\{\begin{array}{l}
\varepsilon^{2} \Delta u-\lambda_{1} u+\mu_{1} u^{3}+\beta u v^{2}=0 \text { in } \Omega \\
\varepsilon^{2} \Delta v-\lambda_{2} v+\mu_{2} v^{3}+\beta u^{2} v=0 \text { in } \Omega \\
u, v>0 \text { in } \Omega, u=v=0 \text { on } \partial \Omega
\end{array}\right.
$$

where $\Omega \subset \mathbb{R}^{N}(N \leq 3)$ is a smooth and bounded domain, $\varepsilon>0$ is a small parameter, $\lambda_{1}, \lambda_{2}, \mu_{1}, \mu_{2}>0$ are positive constants and $\beta \neq 0$ is a coupling constant. We show that there exists an interval $I=\left[a_{0}, b_{0}\right]$ and a sequence of numbers $0<\beta_{1}<\beta_{2}<\ldots<\beta_{n}<\ldots$ such that for any $\beta \in(0,+\infty) \backslash$ $\left(I \cup\left\{\beta_{1}, \ldots, \beta_{n}, \ldots\right\}\right)$, the above problem has a solution such that both $u$ and $v$ develop a spike layer at the innermost part of the domain. Central to our analysis is the nondegeneracy of radial solutions in $\mathbb{R}^{N}$.

\section{INTRODUCTION}

In this paper, we consider the coupled Gross-Pitaevskii equations, i.e., the coupled nonlinear Schrödinger equations,

$$
\left\{\begin{array}{l}
-\sqrt{-1} \frac{\partial}{\partial t} \Phi_{1}=\varepsilon^{2} \Delta \Phi_{1}+\mu_{1}\left|\Phi_{1}\right|^{2} \Phi_{1}+\beta\left|\Phi_{2}\right|^{2} \Phi_{1} \text { for } y \in \Omega, t>0 \\
-\sqrt{-1} \frac{\partial}{\partial t} \Phi_{2}=\varepsilon^{2} \Delta \Phi_{2}+\mu_{2}\left|\Phi_{2}\right|^{2} \Phi_{2}+\beta\left|\Phi_{1}\right|^{2} \Phi_{2} \text { for } y \in \Omega, t>0 \\
\Phi_{j}=\Phi_{j}(y, t) \in \mathbb{C}, j=1,2 \\
\Phi_{j}(y, t)=0 \text { for } y \in \partial \Omega, t>0, j=1,2
\end{array}\right.
$$

where $\epsilon, \mu_{1}, \mu_{2}$ are positive constants, $\Omega$ is a domain in $\mathbb{R}^{N}, N \leq 3$, and $\beta$ is a coupling constant.

System (1.1) arises in many physical problems. When $\Omega$ is a bounded domain, problem (1.1) arises in the Hartree-Fock theory for a double condensate, i.e., a binary mixture of Bose-Einstein condensates in two different hyperfine states $|1\rangle$ and $|2\rangle([19])$. Physically, $\left|\Phi_{1}\right|$ and $\left|\Phi_{2}\right|$ are the corresponding condensate amplitudes, and $\mu_{j}$ and $\beta$ are the intraspecies and interspecies scattering lengths respectively. The sign of the scattering length $\beta$ determines whether the interactions of states $|1\rangle$ and $|2\rangle$ are repulsive or attractive. When $\beta<0$, the interactions of states $|1\rangle$ and $|2\rangle$ are repulsive (34). In contrast, when $\beta>0$, the interactions of states $|1\rangle$ and $|2\rangle$ are attractive. For atoms of the single state $|j\rangle$, when $\mu_{j}>0$, the interactions of the single state $|j\rangle$ are attractive.

Received by the editors August 1, 2006.

2000 Mathematics Subject Classification. Primary 35B40, 35B45; Secondary 35J40.

Key words and phrases. Spike layers, Bose-Einstein condensates, coupled nonlinear Schrödinger equations. 
When $\Omega=\mathbb{R}^{N}$, system (1.1) also arises in the study of incoherent solitons in nonlinear optics. We refer to [29, 30] for experimental results and [2, 7, 21, 22, 23 , for a comprehensive list of references. Physically, the solution $\Phi_{j}$ denotes the $j$-th component of the beam in Kerr-like photorefractive media. The positive constant $\mu_{j}$ is for self-focusing in the $j$-th component of the beam. The coupling constant $\beta$ is the interaction between the first and the second component of the beam. As $\beta>0$, the interaction is attractive, while the interaction is repulsive if $\beta<0$.

In order to obtain solitary wave solutions of the system (1.1), we set $\Phi_{1}(x, t)$ $=e^{\sqrt{-1} \lambda_{j} t} u(x), \Phi_{2}(x, t)=e^{\sqrt{-1} \lambda_{j} t} v(x)$, and the system (1.1) is transformed to an elliptic system given by

$$
\left\{\begin{array}{l}
\varepsilon^{2} \Delta u-\lambda_{1} u+\mu_{1} u^{3}+\beta u v^{2}=0 \text { in } \Omega \\
\varepsilon^{2} \Delta v-\lambda_{2} v+\mu_{2} v^{3}+\beta u^{2} v=0 \text { in } \Omega \\
u, v>0 \text { in } \Omega \\
u=v=0 \text { on } \partial \Omega
\end{array}\right.
$$

where $\Omega \subset \mathbb{R}^{N}(N \leq 3)$ is a smooth and bounded domain, $\varepsilon>0$ is a small parameter, $\lambda_{1}, \lambda_{2}, \mu_{1}, \mu_{2}>0$ are positive constants and $\beta \neq 0$ is a coupling constant.

When $\Omega=\mathbb{R}^{N}$, the existence of the least energy solution to (1.2) is studied in [1, 25, 33]. In [1, 3, 28, 33], the existence of bound states (i.e., solutions to (1.2)) when $\beta>0$ is proved.

In [26], the following result was proved:

Theorem 1.1. There exists a constant $\beta_{0}=\beta_{0}\left(N, \lambda_{1}, \lambda_{2}, \mu_{1}, \mu u_{2}\right) \in\left(0, \sqrt{\mu_{1} \mu_{2}}\right)$ such that the following holds:

(1) For any $\beta \in\left(-\infty, \beta_{0}\right)$ and $\varepsilon$ sufficiently small, (1.2) has a least energy solution $\left(u_{\varepsilon}, v_{\varepsilon}\right)$. Let $P_{\varepsilon}$ be a local maximum point of $u_{\varepsilon}$ and $Q_{\varepsilon}$ be a local maximum point of $v_{\varepsilon}$.

(2) If $0<\beta<\beta_{0}$, then $\left|P_{\varepsilon}-Q_{\varepsilon}\right| / \varepsilon \rightarrow 0$ and

$$
d\left(P_{\varepsilon}, \partial \Omega\right) \rightarrow \max _{P \in \Omega} d(P, \partial \Omega), \quad d\left(Q_{\varepsilon}, \partial \Omega\right) \rightarrow \max _{P \in \Omega} d(P, \partial \Omega) .
$$

Furthermore, $u_{\varepsilon}(x), v_{\varepsilon}(x) \rightarrow 0$ in $C_{l o c}^{1}\left(\bar{\Omega} \backslash\left\{P_{\varepsilon}, Q_{\varepsilon}\right\}\right)$ and let

$$
U_{\varepsilon}(y):=u_{\varepsilon}\left(P_{\varepsilon}+\varepsilon y\right), \quad V_{\varepsilon}(y):=v_{\varepsilon}\left(P_{\varepsilon}+\varepsilon y\right) .
$$

Then as $\varepsilon \rightarrow 0,\left(U_{\varepsilon}, V_{\varepsilon}\right) \rightarrow\left(U_{0}, V_{0}\right)$, which is a least-energy solution of the following problem in $\mathbb{R}^{N}$ :

$$
\left\{\begin{array}{l}
\Delta U_{0}-\lambda_{1} U_{0}+\mu_{1} U_{0}^{3}+\beta U_{0} V_{0}^{2}=0 \text { in } \mathbb{R}^{N} \\
\Delta V_{0}-\lambda_{2} V_{0}+\mu_{2} V_{0}^{3}+\beta U_{0}^{2} V_{0}=0 \text { in } \mathbb{R}^{N}, \\
U_{0}(0)=\max _{y \in \mathbb{R}^{N}} U_{0}(y), V_{0}(0)=\max _{y \in \mathbb{R}^{N}} V_{0}(y), \\
U_{0}, V_{0}>0 \text { in } \mathbb{R}^{N}, U_{0}, V_{0} \rightarrow 0 \text { as }|y| \rightarrow+\infty
\end{array}\right.
$$

(3) If $\beta<0$, then we have

$$
\varphi\left(P_{\varepsilon}, Q_{\varepsilon}\right) \rightarrow \max _{(P, Q) \in \Omega^{2}} \varphi(P, Q)
$$

where

$\varphi(P, Q)=\min \left\{\sqrt{\lambda_{1}}|P-Q|, \sqrt{\lambda_{2}}|P-Q|, \sqrt{\lambda_{1}} d(P, \partial \Omega), \sqrt{\lambda_{2}} d(Q, \partial \Omega)\right\}$.

Furthermore, $u_{\varepsilon}(x), v_{\varepsilon}(x) \rightarrow 0$ in $C_{l o c}^{1}\left(\bar{\Omega} \backslash\left\{P_{\varepsilon}, Q_{\varepsilon}\right\}\right)$, and if we let

$$
U_{\varepsilon}(y):=u_{\varepsilon}\left(P_{\varepsilon}+\varepsilon y\right), \quad V_{\varepsilon}(y):=v_{\varepsilon}\left(Q_{\varepsilon}+\varepsilon y\right)
$$




$$
\begin{aligned}
& \text { then } \\
& U_{\varepsilon}(y) \rightarrow w_{1}(y), \quad V_{\varepsilon}(y) \rightarrow w_{2}(y), \\
& \text { (1.7) } \\
& \text { where } w_{i}(y), i=1,2 \text {, is the unique solution of } \\
& \left\{\begin{array}{l}
\Delta w_{i}-\lambda_{i} w_{i}+\mu_{i} w_{i}^{3}=0 \text { in } \mathbb{R}^{N}, \\
w_{i}(0)=\max _{y \in \mathbb{R}^{N}} w_{i}(y), i=1,2, \quad w_{i}>0 \text { in } \mathbb{R}^{N}, w_{i}(y) \rightarrow 0 \text { as }|y| \rightarrow+\infty .
\end{array}\right.
\end{aligned}
$$

The case when $\beta>\beta_{0}$ was left open. In this paper, we solve this problem by proving the following result:

Theorem 1.2. There exists an interval $I=\left[a_{0}, b_{0}\right] \subset(0,+\infty)$ and a sequence of numbers $\beta_{1}<\beta_{2}<\ldots<\beta_{n}<\ldots$ such that for any $\beta \in(0,+\infty) \backslash\left(I \cup\left\{\beta_{1}, \ldots, \beta_{n}, \ldots\right\}\right)$ and $\varepsilon$ sufficiently small, (1.2) has a solution $\left(u_{\varepsilon}, v_{\varepsilon}\right)$. Let $P_{\varepsilon}$ be a local maximum point of $u_{\varepsilon}$ and $Q_{\varepsilon}$ be a local maximum point of $v_{\varepsilon}$. Then $\left|P_{\varepsilon}-Q_{\varepsilon}\right| / \varepsilon \rightarrow 0$ and

$$
d\left(P_{\varepsilon}, \partial \Omega\right) \rightarrow \max _{P \in \Omega} d(P, \partial \Omega), \quad d\left(Q_{\varepsilon}, \partial \Omega\right) \rightarrow \max _{P \in \Omega} d(P, \partial \Omega) .
$$

Furthermore, $u_{\varepsilon}(x), v_{\varepsilon}(x) \rightarrow 0$ in $C_{l o c}^{1}\left(\bar{\Omega} \backslash\left\{P_{\varepsilon}, Q_{\varepsilon}\right\}\right)$ and let

$$
U_{\varepsilon}(y):=u_{\varepsilon}\left(P_{\varepsilon}+\varepsilon y\right), \quad V_{\varepsilon}(y):=v_{\varepsilon}\left(P_{\varepsilon}+\varepsilon y\right)
$$

then as $\varepsilon \rightarrow 0,\left(U_{\varepsilon}, V_{\varepsilon}\right) \rightarrow\left(U_{0}, V_{0}\right)$, which is a solution of the problem (1.4).

Remark. 1. We can also construct solutions at local maximum points of the distance function.

2. The interval $I$ is almost necessary for existence. In fact, let us suppose $\lambda_{1} \leq \lambda_{2}, \mu_{2}<\mu_{1}$ and $\mu_{2}<\beta<\mu_{1}$. Multiplying the equation (1.2) for $u$ by $v$ and the equation (1.2) for $v$ by $u$, and then integrating by parts and subtracting together, we obtain

$$
\left(\lambda_{2}-\lambda_{1}\right) \int_{\Omega} u v+\int_{\Omega}\left[\left(\mu_{1}-\beta\right) u^{3} v+\left(\beta-\mu_{2}\right) u v^{3}\right]=0
$$

which implies that $u, v \equiv 0$. This implies that there are no solutions to (1.2) if $\lambda_{1} \leq \lambda_{2}, \mu_{2}<\beta<\mu_{1}$.

One attempt at proving Theorem 1.2 is to use the mountain-pass lemma and analyze the mountain-pass solution. The problem is that such a solution may become trivial (i.e., $u=0, v=0$ ) or semitrivial (i.e, $u=0$ or $v=0$ ). Furthermore, there is no simple characterization of mountain-pass solutions when $\beta$ becomes large and also the solutions to (1.4) may not be unique.

Our proof of Theorem 1.2 is by the so-called "Localized Energy Method". That is, we first use a Liapunov-Schmidt method to reduce the problem to a finitedimensional problem, and then use variational methods to find critical points of the reduced finite-dimensional problem. Such a method has been used successfully in many papers for the scalar equations, see e.g. [4], [5], 15, [13], 14], [16], [17] and 24. (In particular, we follow 24.) However, as far as the authors know, this method has never been used for strongly coupled elliptic equations. One of the main difficulties in using this method is the nondegeneracy assumption which is difficult to prove for systems. For single scalar equations, the nondegeneracy can be proved by using the uniqueness of radial solutions (see Appendix $\mathrm{C}$ of 31]). However for systems, the uniqueness of the radial solutions seems out of reach at this moment. Here, we use an idea of the first author in [11] by showing that nondegeneracy holds for (1.4) except for isolated points of $\beta$. More precisely, let $\left(U_{1}, U_{2}\right)$ be a solution 
of (1.4). We say that $\left(U_{1}, U_{2}\right)$ is nondegenerate if the solution set of the linearized equation

$$
\left\{\begin{array}{l}
\Delta \phi_{1}-\lambda_{1} \phi_{1}+3 \mu_{1} U_{1}^{2} \phi_{1}+\beta U_{2}^{2} \phi_{1}+2 \beta U_{1} U_{2} \phi_{2}=0 \\
\Delta \phi_{2}-\lambda_{2} \phi_{2}+3 \mu_{2} U_{2}^{2} \phi_{2}+\beta U_{1}^{2} \phi_{2}+2 \beta U_{1} U_{2} \phi_{1}=0 \\
\left|\phi_{1}\right|+\left|\phi_{2}\right| \leq 1
\end{array}\right.
$$

is exactly $N$-dimensional, namely,

$$
\left(\begin{array}{l}
\phi_{1} \\
\phi_{2}
\end{array}\right)=\sum_{j=1}^{N} a_{j}\left(\begin{array}{c}
\frac{\partial U_{1}}{\partial z_{j}} \\
\frac{\partial U_{2}}{\partial z_{j}}
\end{array}\right)
$$

for some constants $a_{j}$.

The following result is proved in this paper, and contains elements of independent interest.

Theorem 1.3. There exists an interval $I=\left[a_{0}, b_{0}\right] \subset(0,+\infty)$ and a sequence of numbers $\beta_{1}<\beta_{2}<\ldots<\beta_{n}<\ldots$ such that for any $\beta \in(0,+\infty) \backslash\left(I \cup\left\{\beta_{1}, \ldots, \beta_{n}, \ldots\right\}\right)$, problem (1.4) has a nondegenerate solution $\left(U_{1}, U_{2}\right)$.

Remark. 1. The set $I$ is given explicitly in Section 2. See (2.7). As remarked before, there is not always existence if $\beta \in I$.

2. We believe that the set $\left\{\beta_{1}, \beta_{2}, \ldots, \beta_{n}, \ldots\right\}$ is finite. In particular, for $\beta$ large, all solutions to (1.4) are nondegenerate.

3. Our proof of Theorem 1.3 also gives a new proof of existence of solutions to (1.4). Here we use bifurcation analysis, which is different from those of [1, 3] and [33] where variational or topological method is used.

The organization of this paper is as follows. In Section 2 and Section 3, we prove Theorem 1.3. Section 2 contains nondegeneracy in the space of radial functions while Section 3 contains nondegeneracy in the space of nonradial functions. From Section 4 to Section 6 , we apply the localized energy method to prove Theorem 1.2. Section 4 studies a linear problem, Section 5 studies a nonlinear problem and Section 6 completes the proof of Theorem 1.2 ,

\section{Nondegeneracy in the SPACE OF RADIAL FUnCtions}

Let $(u, v)$ be a solution of (1.4). By the moving plane method, as in 35, $u$ and $v$ are both radially symmetric and strictly decreasing, i.e., $u=u(r), v=v(r), u^{\prime}(r)<$ $0, v^{\prime}(r)<0$ for $r \neq 0$. We say that $(u(r), v(r))$ is locally unique if the linearized problem

$$
\left\{\begin{array}{l}
\Delta \phi_{1}-\lambda_{1} \phi_{1}+3 \mu_{1} u^{2} \phi_{1}+\beta v^{2} \phi_{1}+2 \beta u v \phi_{2}=0 \\
\Delta \phi_{2}-\lambda_{2} \phi_{2}+3 \mu_{2} v^{2} \phi_{2}+\beta u^{2} \phi_{2}+2 \beta u v \phi_{1}=0, \\
\phi_{1}=\phi_{1}(r), \phi_{2}=\phi_{2}(r)
\end{array}\right.
$$

admits only trivial decaying solutions. In this section, we prove the following theorem.

Theorem 2.1. There exists $I=\left[a_{0}, b_{0}\right]$ and $\beta_{1}<\beta_{2}<\ldots$ such that problem (1.4) has a locally unique nondegenerate solution for $\beta \notin I_{0} \cup\left\{\beta_{1}, \ldots, \beta_{n}, \ldots\right\}$. 
Before we prove Theorem 2.1 we need some definitions and lemmas. We consider

$$
\left\{\begin{array}{l}
\Delta u-\lambda_{1} u+\mu_{1} u^{3}+\beta u v^{2}=0 \text { in } \mathbb{R}^{N} \\
\Delta v-\lambda_{2} v+\mu_{2} v^{3}+\beta u^{2} v=0 \text { in } \mathbb{R}^{N} \\
u(0)=\max _{y \in \mathbb{R}^{N}} u(y), v(0)=\max _{y \in \mathbb{R}^{N}} v(y) \\
u=u(r), v=v(r)>0 \text { in } \mathbb{R}^{N}, u, v \rightarrow 0 \text { as }|y| \rightarrow+\infty
\end{array}\right.
$$

Note that equation (2.2) admits three trivial solutions

$$
(0,0),(\bar{u}, 0),(0, \bar{v})
$$

where $\bar{u}=w_{1}, \bar{v}=w_{2}$, and $w_{j}$ is the unique solution of (1.7). By a simple scaling

$$
w_{j}(r)=\sqrt{\frac{\lambda_{j}}{\mu_{j}}} w\left(\sqrt{\lambda_{j}} r\right)
$$

where $w$ is the unique solution to (1.7) with $\lambda_{j}=\mu_{j}=1$.

Let us define

$$
a_{0}=\inf _{\phi \in H^{1}} \frac{\int_{\mathbb{R}^{N}}\left(|\nabla \phi|^{2}+\lambda_{2} \phi^{2}\right)}{\int_{\mathbb{R}^{N}} w_{1}^{2} \phi^{2}}, \quad b_{0}=\inf _{\phi \in H^{1}} \frac{\int_{\mathbb{R}^{N}}\left(|\nabla \phi|^{2}+\lambda_{1} \phi^{2}\right)}{\int_{\mathbb{R}^{N}} w_{2}^{2} \phi^{2}} .
$$

Without loss of generality, we may assume that

$$
a_{0} \leq b_{0} \text {. }
$$

We then set

$$
I:=\left[a_{0}, b_{0}\right] .
$$

When $\lambda_{1}=\lambda_{2}$, problem (2.2) admits a bound state of the form

$$
\left(u_{0}, v_{0}\right)=\left(\sqrt{\mu_{1}} c_{1} w_{1}, \sqrt{\mu_{2}} c_{2} w_{2}\right),
$$

where

$$
c_{1}=\sqrt{\frac{\mu_{2}-\beta}{\mu_{1} \mu_{2}-\beta^{2}}}, c_{2}=\sqrt{\frac{\mu_{1}-\beta}{\mu_{1} \mu_{2}-\beta^{2}}}
$$

as long as $\left(\mu_{1}-\beta\right)\left(\mu_{2}-\beta\right)>0$, i.e., $\beta \notin\left[\min \left(\mu_{1}, \mu_{2}\right), \max \left(\mu_{1}, \mu_{2}\right)\right]$. Note also that by a simple scaling

$$
a_{0}=\mu_{1} \inf _{\phi \in H^{1}} \frac{\int_{\mathbb{R}^{N}}\left(|\nabla \phi|^{2}+\phi^{2}\right)}{\int_{\mathbb{R}^{N}} w^{2} \phi^{2}}=\mu_{1} .
$$

(See the proof of Lemma 2.2 below.) Similarly $b_{0}=\mu_{2}$. Thus in this case $I=$ $\left[\min \left(\mu_{1}, \mu_{2}\right), \max \left(\mu_{1}, \mu_{2}\right)\right]$.

Our next lemma says that this solution is nondegenerate in the space of radial functions. (Note that in this case, by the Remark after Theorem 1.2 there is no existence to (1.4) for $\beta \in\left[\min \left(\mu_{1}, \mu_{2}\right), \max \left(\mu_{1}, \mu_{2}\right)\right]$.)

Lemma 2.2. Let $\lambda_{1}=\lambda_{2}$ and $\beta \notin\left[\min \left(\mu_{1}, \mu_{2}\right), \max \left(\mu_{1}, \mu_{2}\right)\right]$. Then the solution $\left(u_{0}, v_{0}\right)$ defined by (2.8) is nondegenerate in the space of radial functions.

Proof. We just need to study the eigenvalue problem (2.1) associated with $\left(u_{0}, v_{0}\right)$. Let $\lambda_{1}=\lambda_{2}=\lambda_{0}$. Without loss of generality, we may assume that $\lambda_{0}=1$. Then $\left(u_{0}, v_{0}\right)=\left(c_{1} w, c_{2} w\right)$ and (2.1) becomes

$$
\left\{\begin{array}{l}
\Delta \phi_{1}-\phi_{1}+\left(3 \mu_{1} c_{1}^{2}+\beta c_{2}^{2}\right) w^{2} \phi_{1}+2 \beta c_{1} c_{2} w^{2} \phi_{2}=0, \\
\Delta \phi_{2}-\phi_{2}+\left(3 \mu_{2} c_{2}^{2}+\beta c_{1}^{2}\right) \phi_{2}+2 \beta c_{1} c_{2} w^{2} \phi_{1}=0, \\
\phi_{1}=\phi_{1}(r), \phi_{2}=\phi_{2}(r) .
\end{array}\right.
$$


By an orthonormal transformation, (2.9) can be transformed to two single equations

$$
\begin{gathered}
\Delta \Phi_{1}-\Phi_{1}+3 w^{2} \Phi_{1}=0, \Phi_{1}=\Phi_{1}(r), \\
\Delta \Phi_{2}-\Phi_{2}+\left(3-2 \beta\left(c_{1}^{2}+c_{2}^{2}\right)\right) w^{2} \Phi_{2}=0, \Phi_{2}=\Phi_{2}(r) .
\end{gathered}
$$

Note that the eigenvalues of

$$
\Delta \Phi-\Phi+\nu w^{2} \Phi=0, \Phi \in H^{1}\left(\mathbb{R}^{N}\right)
$$

are

$$
\nu_{1}=1, \nu_{2}=\ldots=\nu_{N+1}=3, \nu_{N+2}>3,
$$

where the eigenfunction corresponding to $\nu_{1}$ is $c w$, and the eigenfunctions corresponding to $\nu_{2}$ are spanned by $\frac{\partial w}{\partial z_{j}}, j=1, \ldots, N$. (See Lemma 4.1 of [36].)

Hence $\Phi_{1}=0 . \Phi_{2}=0$ unless $3-2 \beta\left(c_{1}^{2}+c_{2}^{2}\right)=1$. If $3-2 \beta\left(c_{1}^{2}+c_{2}^{2}\right)=1$, then we have $\beta=\mu_{1}=\mu_{2}$, since $\mu_{1} c_{1}^{2}+\beta c_{2}^{2}=\mu_{2} c_{2}^{2}+\beta c_{1}^{2}=1$. This is impossible since $\beta \notin\left[\min \left(\mu_{1}, \mu_{2}\right), \max \left(\mu_{1}, \mu_{2}\right)\right]$.

In conclusion, we derive that $\Phi_{1}=\Phi_{2}=0$ and hence $\phi_{1}=\phi_{2}=0$.

Next we show that $a_{0}$ or $b_{0}$ is a bifurcation point.

Lemma 2.3. When $\beta=a_{0},(u, v)=(\bar{u}, 0)$ or $\beta=b_{0},(u, v)=(0, \bar{v})$, the linearized problem (2.1) has exactly a one-dimensional set of solutions.

Proof. It is easy to see that $a_{0}$ can be attained by a radially symmetric and positive function denoted by $\bar{\psi}_{0}$ (normalized so that $\left.\bar{\psi}_{0}(0)=1\right)$. Let $\beta=a_{0},(u, v)=(\bar{u}, 0)$. Then (2.1) becomes

$$
\left\{\begin{array}{l}
\Delta \phi_{1}-\lambda_{1} \phi_{1}+3 \mu_{1} \bar{u}^{2} \phi_{1}=0, \\
\Delta \phi_{2}-\lambda_{2} \phi_{2}+a_{0} \bar{u}^{2} \phi_{2}=0, \\
\phi_{1}=\phi_{1}(r), \phi_{2}=\phi_{2}(r) .
\end{array}\right.
$$

Note that the two equations in (2.14) are decoupled. By the same argument as those of Lemma 2.2, we have $\phi_{1}=0$. On the other hand, by the definition of $a_{0}$, we see that $\phi_{2}$ is the principal eigenfunction, and hence $\phi_{2}=c \bar{\psi}_{0}$ for some $c>0$.

Henceforth, we may assume that

$$
\lambda_{1} \neq \lambda_{2} .
$$

We will work on the space $E=C_{r, 0}\left(\mathbb{R}^{N}\right) \times C_{r, 0}\left(\mathbb{R}^{N}\right)$, where $C_{r, 0}\left(\mathbb{R}^{N}\right)$ denotes the space of continuous radial functions vanishing at $\infty$. We can write the system (2.2) on $E$ as

$$
u=\left(-\Delta+\lambda_{1}\right)^{-1}\left(\mu_{1} u^{3}+\beta u v^{2}\right), \quad v=\left(-\Delta+\lambda_{2}\right)^{-1}\left(\mu_{2} v^{3}+\beta u^{2} v\right) .
$$

We also need the following lemma.

Lemma 2.4. (1) For each fixed $\beta>0$, there is a $C_{\beta}>0$ such that

$$
\|u\|_{L^{\infty}\left(\mathbb{R}^{N}\right)}+\|v\|_{L^{\infty}\left(\mathbb{R}^{N}\right)} \leq C_{\beta}
$$

for any nonnegative solution $(u, v)$ of (2.16).

(2) Let $\beta$ be bounded. Then the set of nonnegative solutions of (2.16) is compact in $E$. 
Proof. (1). We proceed by contradiction, assuming that there is a sequence of solutions $\left(u_{n}, v_{n}\right)$ to (1.4) with

$$
\max _{x \in \mathbb{R}^{N}} u_{n}(x)+\max _{x \in \mathbb{R}^{N}} v_{n}(x) \rightarrow+\infty \quad \text { as } n \rightarrow \infty .
$$

We follow a blow up procedure introduced by Gidas and Spruck 20, for scalar equations. Since the method is standard, we only sketch the argument. Without loss of generality, we may assume that

$$
M_{n}:=u_{n}(0)=\max _{x \in \mathbb{R}^{N}} u_{n}(x) \geq v_{n}(0)=\max _{x \in \mathbb{R}^{N}} v_{n}(x) .
$$

Now we perform a rescaling, setting $x=\frac{y}{M_{n}}$ and defining functions $U_{n}, V_{n}: \mathbb{R}^{N} \rightarrow$ $\mathbb{R}$ by

$$
U_{n}(y)=\frac{u_{n}\left(\frac{y}{M_{n}}\right)}{M_{n}}, \quad V_{n}(y)=\frac{v_{n}\left(\frac{y}{M_{n}}\right)}{M_{n}} \quad \text { for } y \in \mathbb{R}^{N}
$$

Then

$$
1:=\max _{y \in \mathbb{R}^{N}} U_{n}(y) \geq \max _{y \in \mathbb{R}^{N}} V_{n}(y),
$$

and $\left(U_{n}, V_{n}\right)$ solves the rescaled problem

$$
\begin{cases}-\Delta U_{n}=\mu_{1} U_{n}^{3}+\beta U_{n} V_{n}^{2}-\frac{\lambda_{1}}{M_{n}^{2}} U_{n} & \text { in } \mathbb{R}^{N}, \\ -\Delta V_{n}=\mu_{1} V_{n}^{3}+\beta V_{n} U_{n}^{2}-\frac{\lambda_{2}}{M_{n}^{2}} V_{n} & \text { in } \mathbb{R}^{N} .\end{cases}
$$

Passing to a subsequence if necessary, we see that $\left(U_{n}, V_{n}\right) \rightarrow\left(U_{0}, V_{0}\right)$, which is a nontrivial and nonnegative bounded radial solution of

$$
-\Delta u=\mu_{1} u^{3}+\beta u v^{2},-\Delta v=\mu_{2} v^{3}+\beta v u^{2} .
$$

If $u$ is nonnegative, then we have $-\Delta u \geq \mu_{1} u^{3}$ on $\mathbb{R}^{N}$ which implies $u \equiv 0$ by standard results (if $N \leq 3$ ). See [20]. Similarly if $v$ is nontrivial, we also obtain a contradiction. Hence we have the a priori bound (2.17).

(2) We now show that the set of nonnegative solutions to (2.16) cannot become noncompact in $E$ for bounded $\beta$ (while it remains nonnegative and bounded in $E)$. To do this, we note that a bounded set $T$ in $C_{r, 0}\left(\mathbb{R}^{N}\right)$ is precompact (that is, it has compact closure) if and only if it is equicontinuous on bounded sets, and given $\epsilon>0$, there is a $\mu>0$ such that $|u(r)| \leq \epsilon$ if $r \geq \mu$ and $u \in T$. (This can most easily be seen if we identify $C_{r, 0}\left(\mathbb{R}^{N}\right)$ with $\{w \in C[0,1] ; w(1)=0\}$ by mapping $[0, \infty)$ onto $[0,1]$ with $\infty$ corresponding to 1 and using the Arzela-Ascoli compactness theorem.) Now our branch is bounded in $C^{2}\left(\mathbb{R}^{N}\right)$ for bounded $\beta$. Thus we can only have trouble with the second condition, i.e., if there exists $\alpha>0$ and $r_{i} \rightarrow+\infty$ and solutions $\left(u_{i}, v_{i}\right)$ to (2.16) with $u_{i}\left(r_{i}\right)+v_{i}\left(r_{i}\right)=\alpha$. By changing the origin to $r_{i}$ and passing to the limit, we will obtain a nontrivial solution $(u, v)$ on $\mathbb{R}$ of the following problem:

$$
-u^{\prime \prime}=-\lambda_{1} u+\mu_{1} u^{3}+\beta u v^{2}, \quad-v^{\prime \prime}=-\lambda_{2} v+\mu_{2} v^{3}+\beta v u^{2}
$$

with $u(0)+v(0)=\alpha, u, v \geq 0$ and bounded. Moreover $u$ and $v$ must be decreasing on $\mathbb{R}$ since nonnegative solutions of (2.2) are decreasing on $[0, \infty)$. Hence $u$ and $v$ have limits $u_{+}, v_{+}$at $\infty$ (and limit $\left(u_{-}, v_{-}\right)$at $\left.-\infty\right)$. Thus $\left(u_{-}, v_{-}\right)$and $\left(u_{+}, v_{+}\right)$both solve $\lambda_{1} u=\mu_{1} u^{3}+\beta u v^{2}, \lambda_{2} v=\mu_{2} v^{3}+\beta u^{2} v$. If we choose $\alpha$ small, $u_{+}+v_{+} \leq \alpha$, and hence $u_{+}=v_{+}=0$. If $u$ does not vanish identically, $u_{-}>0$ and $\lambda_{1}=u_{-}^{2}+\beta v_{-}^{2}$. 
Hence $u\left(-\lambda_{1}+u^{2}+\beta v^{2}\right)<0$ on $\mathbb{R}$ (by the decreasing properties of $u$ and $v$ ) and hence $u^{\prime \prime}>0$ on $\mathbb{R}$. Thus $u$ is strictly convex and bounded on $\mathbb{R}$ which is impossible. Hence compactness of nonnegative solutions to (2.16) holds.

We proceed to prove Theorem 2.1.

When $\beta=0$, it is known that there exists a unique positive solution $(\bar{u}, \bar{v})$ which is the only nonnegative solution for $\beta=0$ (except for $(\bar{u}, 0)$ and $(0, \bar{v})$ ). Note that $(\bar{u}, \bar{v})$ is nondegenerate for $\beta=0$ in $E$ since the system (2.16) is diagonal, and we can use the results for scalar equations. The operator obtained by linearizing the right hand side of (2.16) at $(\bar{u}, \bar{v})$ is compact on $E$, since $(u, v)$ decays. (Note that the nonlinear mapping itself is not completely continuous on $E$.) Hence we can use the degree theory for $C^{2}$ Fredholm maps ([10]). (Note that the map is smooth in $E$ and a number of authors have considered similar degrees.) For the moment, we need to consider changing-sign solutions of (2.16). By a slight variant of the theory in Section 1 of [10], there is a connected set $\tilde{C}$ of solutions of (2.16) in $E \times \mathbb{R}$ with $\beta \geq 0$ starting at $(\bar{u}, \bar{v})$ for $\beta=0$, while it is noncompact in $E \times \mathbb{R}$ or contains another solution for $\beta=0$. (This is a standard Rabinowitz type continuation result where we start from a nondegenerate solution rather than from the zero solution.) Moreover, $\tilde{C}$ can only leave the cone of nonnegative functions at $(\bar{u}, 0)$ for $\beta=a_{0}$ or at $(0, \bar{v})$ for $\beta=b_{0}$, where $a_{0} \leq b_{0}$ are defined by (2.5). To see this, suppose that $\left(u_{n}, v_{n}\right)$ are solutions of (2.16) such that $\left(u_{n}, v_{n}, \beta_{n}\right)$ converges to $\left(u_{0}, v_{0}, \beta_{0}\right)$ in $E \times \mathbb{R}$, where $u_{n}$ changes sign and $u_{0}$ is positive on $(0, \infty)$. Then $u_{n}\left(r_{n}\right)$ must be small and $r_{n}$ must be large whenever $u_{n}\left(r_{n}\right) \leq 0$, for large $n$. Thus any component $Z_{n}$ of $\left\{r: u_{n}(r)<0\right\}$ must be where $r$ is large and $\left|u_{n}\right|+v_{n} \mid$ is small. (Recall that $\left|u_{0}\right|+\left|v_{0}\right| \rightarrow 0$ as $r \rightarrow+\infty$.) Hence $-\lambda_{1}+\mu_{1} u_{n}^{2}+\beta v_{n}^{2}$ is negative on $Z_{n}$ and thus, by the equation for $u_{n}, \Delta u_{n}<0$ on $Z_{n}$. This contradicts the fact that $u_{n}$ must have a minimum on $Z_{n}$. If we only assumed that $u_{0}$ was nonnegative on $[0, \infty)$, the maximum principle applied to the equation for $u_{0}$ implies that either $u_{0} \equiv 0$ or $u_{0}(r)>0$ on $[0, \infty)$. Hence we see that $\tilde{C}$ can only leave the cone of nonnegative functions if there exist solutions $\left(u_{n}, v_{n}\right)$ for $\beta=\beta_{n}$ such that $\left(u_{n}, v_{n}\right) \rightarrow\left(0, v_{0}\right)$ in $E$ and $\beta \rightarrow \beta_{0}$ as $n \rightarrow \infty$, where $u_{n}, v_{n}$ and $v_{0}$ are positive solutions on $[0, \infty)$. (Note that $\left\|u_{n}\right\|_{L^{\infty}}$ and $\left\|v_{n}\right\|_{L^{\infty}}$ cannot both be small from the equation (2.16).) By the first equation, we easily see that $\frac{u_{n}}{\left\|u_{n}\right\|_{L} \infty}$ must converge to a positive decreasing solution of $-\Delta h=\left(-\lambda_{1}+\beta_{0} v_{0}^{2}\right) h$ on $[0, \infty)$, at least through a subsequence. $h$ must be nontrivial because $u_{n}$ has its maximum at zero. Hence $\beta_{0}=b_{0}$. Thus our claim is proved and the branch $\tilde{C}$ continues up to $\min \left(a_{0}, b_{0}\right)=a_{0}$ (where we can assume $a_{0} \leq b_{0}$ without loss of generality).

We now note that our nonlinearity is easily seen to be real analytic on $E$ (in fact it is polynomial). Since $\tilde{C}$ starts from $\beta=0$ where there is a unique and nondegenerate positive solution, Theorem 1 of $\left[11\right.$ implies that for all $\beta$ in $\left[0, a_{0}\right)$ except at isolated points, there are an odd number of nondegenerate positive solutions in the component $\tilde{C}$ (nondegenerate in $E$ ). There is one minor point here. The results in 12 imply that $C$ is locally connected and hence we can choose an open neighborhood $\mathcal{U}$ of $\tilde{C}$ in $E \times \mathbb{R}$ containing no other solutions of our equation except those in $\tilde{C}$. In fact, the exceptional points cannot accumulate at $a_{0}$ as we see below. Now by Lemma 2.3, it is easy to see that at $\beta=a_{0}$, there is bifurcation from a simple eigenvalue from the solution $(\bar{u}, 0)$ (which is a solution for all $\beta>0$ ) in the sense of $[8]-9]$. More precisely, the other solutions near $\left(\bar{u}, 0, a_{0}\right)$ form a real 
analytic $\operatorname{arc} u=u(\alpha), v=\alpha(\tilde{h}+\gamma(\alpha)), \beta=\phi(\alpha)$ for $\alpha$ small, where $\alpha$ is a scalar variable, $u(0)=\bar{u}, \gamma(0)=0, \phi(0)=a_{0}$ and $v(\alpha)$ is in a complement to the span $\tilde{h}$ in $C_{r, 0}\left(\mathbb{R}^{N}\right)$. Here $\tilde{h}>0$ is the principal eigenfunction of $-\Delta \tilde{h}=-\lambda_{2} \tilde{h}+\beta \bar{u}^{2} \tilde{h}$. There is an analogous bifurcation at $(0, \bar{v})$ at $\beta=b_{0}$, where in this case $\beta=\psi(\tilde{\alpha})$ with $\psi$ real analytic, $\psi(0)=b_{0}$ and $\tilde{\alpha}$ is a scalar parameter. We will prove below that either $\phi^{\prime}(\alpha) \neq 0$ for small nonzero $\alpha$ and $\psi^{\prime}(\tilde{\alpha}) \neq 0$ for small nonzero $\tilde{\alpha}$, or $\phi$ and $\psi$ are constant functions $a_{0}=b_{0}$ and there is an $\operatorname{arc} A$ of positive solutions of (2.16) for $\beta=a_{0}$ joining $(\bar{u}, 0)$ to $(0, \bar{v})$. We refer to this as Claim $\mathbf{A}$ and defer its proof to the end of the proof of Theorem 2.1. We refer to the second possibility as the exceptional case. If $\phi^{\prime}(\alpha) \neq 0$ for small nonzero $\alpha$, Theorem 1.17 in [8] (or by [10]) implies $(u(\alpha), v(\alpha))$ is a nondegenerate solution for $\beta=\phi(\alpha)$ for small non-zero $\alpha$, and an analogous result holds for $\beta=\psi(\tilde{\alpha})$. If $\phi(\alpha)<a_{0}$ for small positive $\alpha$, we have a nondegenerate solution for $\beta \in\left(a_{0}, a_{0}-\delta\right)$. If $\phi(\alpha)>a_{0}$, for small positive $\alpha$, the argument in Theorem 1 of [11] applies all the way to $a_{0}$, and we have our claim.

If $(\bar{u}, 0)$ and $(0, \bar{v})$ do not belong to the closure of $\tilde{C}$, Theorem 1 in [1] implies that we have a nondegenerate positive solution for all $\beta>0$ except at isolated points. If both $(\bar{u}, 0)$ and $(0, \bar{v})$ belong to the closure of $\tilde{C}$ and we are not in the exceptional case, Theorem 1 in 11 together with our remarks above implies that (except for isolated values of $\beta$ ) the number of nondegenerate positive solutions must change by 1 as $\beta$ crosses $a_{0}$ and by 1 as $\beta$ crosses $b_{0}$ (and by an even number as $\beta$ crosses $a_{0}$ if $a_{0}=b_{0}$ ). Thus for $\beta>b_{0}$, there are an odd number of nondegenerate positive solutions (except for isolated $\beta$ ). If $(\bar{u}, 0)$ is not in the closure of $\tilde{C}$ but $(0, \bar{v})$ is in the closure of $\tilde{C}$, the branch $\tilde{C}_{1}$ of positive solutions coming out of $(\bar{u}, 0)$ at $\beta=a_{0}$ must have an even number of nondegenerate positive solutions for $\beta<a_{0}$ (as usual except for isolated $\beta$ ) since $\tilde{C}_{1}$ does not continue to $\beta=0$. (Note that the only positive solution for $\beta=0$ lies in $\tilde{C}$.) Thus $\tilde{C}_{1}$ must have an odd number of nondegenerate positive solutions for all $\beta>a_{0}$ (as usual except for isolated $\beta$ ), and we are finished. A similar argument is valid if $(0, \bar{v})$ is in the closure of $\tilde{C}$ but $(\bar{u}, 0)$ is not. Finally in the exceptional case, the only solutions leaving the set of positive solutions lie in the arc $A$ which consists of degenerate solutions (since each is nonisolated). These do not affect the argument in the proof of Theorem 1 in [11, and so, once again there is a positive nondegenerate solution for all $\beta>0$ except for isolated $\beta$.

Lastly, we prove Claim A. First, note that, by real analyticity, either $\phi$ is constant or $\phi^{\prime}(\alpha) \neq 0$ for small nonzero $\alpha$. Thus if the first possibility of Claim A fails, then $\phi$ is constant. By the proof of Theorem 1 in [11, $\tilde{C}=\bigcup_{i=1}^{\infty} D_{i}$, where $D_{i}$ for $i \geq 2$ is an $i$-dimensional manifold, $D_{1}$ is closed and the $D_{i}$ 's are disjoint. Since the solution coming out of $(\bar{u}, 0)$ at $\beta=a_{0}$ is locally an arc, this must lie in $D_{1}$. By the theory in [11] and [12, at any point $(z, \mu)$ of $D_{1}, D_{1}$ is locally a finite union of closed $\operatorname{arcs} W_{i}, i=1, \ldots, k$, which intersect only at $(z, \mu),(z, \mu)$ is an interior point of each arc $W_{i}$ and each $W_{i}$ can either be parametrized by $\beta$ (for $\beta$ in $(\mu-\delta, \mu+\delta)$ for $\delta>0)$ or $W_{i}$ lies in $\beta=\mu$. (Different $W_{i}$ can satisfy a different one of these alternatives.) By this and compactness, we see that $\mathcal{T}$-the set of nonnegative solutions in $D_{1} \cap\left\{\beta=a_{0}\right\}$-consists of a finite number of points and a finite number of disjoint arcs joining these points, i.e., a finite graph with an even number of edges at each vertex except at $(\bar{u}, 0)$ (and $(0, \bar{v})$ if $\left.b_{0}=a_{0}\right)$. By elementary graph theory, this is impossible if $a_{0} \neq b_{0}$ and, if $a_{0}=b_{0}$, there is an 
arc in $\mathcal{T}$ joining $(\bar{u}, 0)$ to $(0, \bar{v})$. Since this implies $\psi$ is constant, we have proved Claim A and hence proved Theorem 2.1 .

\section{Nondegeneracy in the general CaSe}

Let $(u, v)$ be the solution of (1.4). In this section, we show that for the linearization of equation (1.4) around the solution $(u, v)$, the nonradial part of the kernel has exactly dimension $\mathrm{N}$ (thus comprising exactly the translational modes). This is summarized as follows:

Theorem 3.1. Suppose that $(\phi, \psi) \in H^{2}\left(\mathbb{R}^{N}\right) \times H^{2}\left(\mathbb{R}^{N}\right)$ satisfies the following eigenvalue problem:

$$
\left\{\begin{array}{l}
\Delta \phi-\lambda_{1} \phi+3 \mu_{1} u^{2} \phi+\beta v^{2} \phi+2 \beta u v \psi=0 \\
\Delta \psi-\lambda_{2} \psi+3 \mu_{2} v^{2} \psi+\beta u^{2} \psi+2 \beta u v \phi=0
\end{array}\right.
$$

where $\beta \neq 0$. Then

$$
\left(\begin{array}{c}
\phi \\
\psi
\end{array}\right) \in \operatorname{span}\left\{\left(\begin{array}{c}
\frac{\partial u}{\partial z_{j}} \\
\frac{\partial v}{\partial z_{j}}
\end{array}\right), j=1, \ldots, N\right\} \cup\left\{\left(\begin{array}{c}
\phi \\
\psi
\end{array}\right) \mid \begin{array}{l}
\phi=\phi(r), \psi=\psi(r), \\
(\phi, \psi) \text { satisfies (2.1) }
\end{array}\right\} .
$$

Proof. We first recall that the eigenvalues of $\Delta_{S^{N}}$ are given by

$$
\mu_{1}=0, \mu_{2}=\ldots=\mu_{N+1}=N-1, \mu_{N+1}<\mu_{N+2}, \ldots .
$$

Let $e_{i}(\theta)$ be the corresponding eigenfunctions, i.e.,

$$
\Delta_{S^{N}} e_{i}+\mu_{i} e_{i}=0, \quad i=1,2, \ldots
$$

For any solution $(\phi, \psi)$ of (3.1) set

$$
\phi_{i}(r)=\int_{S^{N}} \phi(r, \theta) e_{i}(\theta) d \theta, \quad \psi_{i}(r)=\int_{S^{N}} \psi(r, \theta) e_{i}(\theta) d \theta .
$$

Recall that

$$
\Delta \phi=\Delta_{r} \phi+\frac{\Delta_{S^{N}} \phi}{r^{2}}
$$

We obtain the following system:

$$
\left\{\begin{array}{l}
\Delta \phi_{i}-\lambda_{1} \phi_{i}-\frac{\mu_{i}}{r^{2}} \phi_{i}+3 \mu_{1} u^{2} \phi_{i}+\beta v^{2} \phi_{i}+2 \beta u v \psi_{i}=0, \\
\Delta \psi_{i}-\lambda_{2} \psi_{i}-\frac{\mu_{i}}{r^{2}} \psi_{i}+3 \mu_{2} v^{2} \psi_{i}+\beta u^{2} \psi_{i}+2 \beta u v \phi_{i}=0 .
\end{array}\right.
$$

The proof will be finished by showing the following claims.

Claim 1. If $i \geq N+2$, then $\phi_{i} \equiv \psi_{i} \equiv 0$.

Suppose this is not the case. We first multiply (3.4) by $u^{\prime}$ and $v^{\prime}$, respectively, where ${ }^{\prime}=\frac{\partial}{\partial r}$, and integrate over the ball $B_{r}$ centered at the origin with radius $r$. Note that

$$
\left\{\begin{array}{l}
\Delta u^{\prime}-\lambda_{1} u^{\prime}+3 \mu_{1} u^{2} u^{\prime}+\beta v^{2} u^{\prime}+2 \beta u v u^{\prime}=\frac{N-1}{r^{2}} u^{\prime} \\
\Delta v^{\prime}-\lambda_{2} v^{\prime}+3 \mu_{2} v^{2} v^{\prime}+\beta u^{2} v^{\prime}+2 \beta u v v^{\prime}=\frac{N-1}{r^{2}} v^{\prime}
\end{array}\right.
$$


Integration by parts gives

$$
\begin{array}{r}
0=\int_{B_{r}}\left(\Delta \phi_{i}-\lambda_{1} \phi_{i}-\frac{\mu_{i}}{r^{2}} \phi_{i}+3 \mu_{1} u^{2} \phi_{i}+\beta v^{2} \phi_{i}\right) u^{\prime}+\int_{B_{r}} 2 \beta u v \psi_{i} u^{\prime} \\
0=\int_{\partial B_{r}}\left(u^{\prime} \phi_{i}^{\prime}-\phi_{i} u^{\prime \prime}\right)+\int_{B_{r}} \frac{N-1-\mu_{i}}{r^{2}} u^{\prime} \phi_{i} \\
-\int_{B_{r}} 2 \beta u v v^{\prime} \phi_{i}+\int_{B_{r}} 2 \beta u v u^{\prime} \psi_{i} .
\end{array}
$$

Similarly, we get

$$
\begin{gathered}
0=\int_{\partial B_{r}}\left(v^{\prime} \psi_{i}^{\prime}-\psi_{i} v^{\prime \prime}\right)+\int_{B_{r}} \frac{N-1-\mu_{i}}{r^{2}} v^{\prime} \psi_{i} \\
-\int_{B_{r}} 2 \beta u v u^{\prime} \psi_{i}+\int_{B_{r}} 2 u v v^{\prime} \phi_{i} .
\end{gathered}
$$

Adding (3.6) and (3.7) we get

$$
\begin{array}{r}
0=\int_{\partial B_{r}}\left(u^{\prime} \phi_{i}^{\prime}-\phi_{i} u^{\prime \prime}\right)+\int_{\partial B_{r}}\left(v^{\prime} \psi_{i}^{\prime}-\psi_{i} v^{\prime \prime}\right) \\
+\int_{B_{r}} \frac{N-1-\mu_{i}}{r^{2}}\left(u^{\prime} \phi_{i}+v^{\prime} \psi_{i}\right)=I_{1}(r)+I_{2}(r)+I_{3}(r),
\end{array}
$$

where $I_{j}(r), j=1,2,3$, are defined by the last equality. We now choose appropriate $r$ and estimate each of the terms $I_{j}(r)$. By definition we have

$$
\phi_{i}^{\prime}(0)=\psi_{i}^{\prime}(0)=0 .
$$

Without loss of generality we assume that there is some $r_{1}>0$ such that $\phi_{i}(r)<0$ for $0<r<r_{1}$ and $\phi_{i}\left(r_{1}\right)=0$. (We choose $r_{1}=\infty$ if $\phi_{i}<0$ in $(0, \infty)$.) Note that by standard ODE theory $\phi_{i}^{\prime}\left(r_{1}\right)>0$.

We claim that $\psi_{i}(r)<0$ for $r$ small. Suppose this is not the case. Let $\psi_{i}(r)>0$ for $r \in\left(0, r_{2}\right)$ for some $r_{2}>0$. If $r_{2}<r_{1}$, then we have

$$
\begin{gathered}
\int_{\partial B_{r_{2}}}\left(v^{\prime} \psi_{i}^{\prime}-\psi_{i} v^{\prime \prime}\right) \geq 0, \\
\int_{B_{r_{2}}} \frac{N-1-\mu_{i}}{r^{2}} v^{\prime} \psi_{i}>0, \\
\int_{B_{r_{2}}} 2 \beta u v u^{\prime} \psi_{i}<0, \\
\int_{B_{r_{2}}} 2 u v v^{\prime} \phi_{i}>0 .
\end{gathered}
$$

Adding all the above together, we obtain a contradiction to (3.7).

If $r_{2} \geq r_{1}$, we use (3.6) to get a contradiction.

Therefore $\psi_{i}(r)<0$ for $r$ small. This implies that there is some $r_{2}>0$ such that $\psi_{i}(r)<0$ for $0<r<r_{2}$ and $\phi_{i}\left(r_{2}\right)=0$. (We choose $r_{2}=\infty$ if $\psi_{i}<0$ in $(0, \infty)$.) Note that necessarily $\psi_{i}^{\prime}\left(r_{2}\right)>0$.

From now on we distinguish three different cases. 
Case 1.1. $r_{1}=r_{2}$.

Set $r=r_{1}=r_{2}$. We easily calculate

$$
I_{1}(r)<0, \quad I_{2}(r)<0, \quad I_{3}(r)<0 .
$$

By (3.8) this gives a contradiction.

Case 1.2. $r_{2}<r_{1}$.

We easily calculate $I_{3}\left(r_{2}\right)<0$ and $I_{2}\left(r_{2}\right)<0$. It is more difficult to evaluate $I_{1}\left(r_{2}\right)$. We define

$$
\Phi(r)=r^{N-1} \phi_{i}^{\prime}(r) u^{\prime}(r)-r^{n-1} u^{\prime \prime}(r) \phi_{i}(r) .
$$

Then for $r_{2}<r<r_{1}$,

$$
\Phi^{\prime}(r)=\left(r^{N-1} \phi_{i}^{\prime}\right)^{\prime} u^{\prime}-\left(r^{n-1} u^{\prime \prime}\right)^{\prime} \phi_{i} .
$$

Now we use

$$
\begin{gathered}
\frac{1}{r^{N-1}}\left(r^{N-1} \phi_{i}^{\prime}\right)^{\prime}-\lambda_{1} \phi_{i}+3 \mu_{1} u^{2} \phi_{i}+\beta v^{2} \phi_{i}+2 \beta u v \psi_{i}=\frac{\mu_{i}}{r^{2}} \phi_{i}, \\
\frac{1}{r^{N-1}}\left(r^{N-1} u^{\prime \prime}\right)^{\prime}-\lambda_{1} u^{\prime}+3 \mu_{1} u^{2} u^{\prime}+\beta v^{2} u^{\prime}+2 \beta u v v^{\prime}=\frac{N-1}{r^{2}} u^{\prime},
\end{gathered}
$$

and get

$$
\Phi^{\prime}(r)=r^{N-3}\left(\mu_{i}-(N-1)\right) \phi_{i} u^{\prime}-2 r^{n-1} \beta u v u^{\prime} \psi_{i}+2 \beta r^{N-1} u v v^{\prime} \phi_{i}>0 .
$$

Here we have used the fact that for $r_{2}<r<r_{1}, \psi_{i}(r)>0$. In fact, if there is $r_{2}<r_{3}<r_{1}$ such that $\psi(r)>0, \psi\left(r_{3}\right)=0$. Then similar to before, we use (3.7) to deduce a contradiction.

Putting these two facts together we conclude

$$
\begin{aligned}
0 & >r_{1}^{N-1}\left(u^{\prime}\left(r_{1}\right) \phi_{i}^{\prime}\left(r_{1}\right)-\phi_{i}\left(r_{1}\right) u^{\prime \prime}\left(r_{1}\right)\right) \\
& =\Phi\left(r_{1}\right)>\Phi\left(r_{2}\right)=\frac{1}{\left|S^{N-1}\right|} I_{1}\left(r_{2}\right) .
\end{aligned}
$$

By (3.8) this gives a contradiction.

Case 1.3. $r_{1}<r_{2}$.

The proof in this case is similar to Case 1.2. We omit the details.

In conclusion, we have proved that for $i \geq N+2, \phi_{i}(r)=\psi_{i}(r) \equiv 0$. This proves Claim 1.

Claim 2. For $i=2, \ldots, N+1,\left(\phi_{j}, \psi_{j}\right)=c_{j}\left(u^{\prime}, v^{\prime}\right)$ for some constant $c_{j}$.

We have to show that the solution set of

$$
\left\{\begin{array}{l}
\Delta \phi_{i}-\lambda_{1} \phi_{i}+3 \mu_{1} u^{2} \phi_{i}+\beta v^{2} \phi_{i}+2 \beta u v \psi_{i}=\frac{N-1}{r^{2}} \phi_{i} \\
\Delta \psi_{i}-\lambda_{2} \psi_{i}+3 \mu_{2} v^{2} \psi_{i}+\beta u^{2} \psi_{i}+2 \beta u v \phi_{i}=\frac{N-1}{r^{2}} \psi_{i} \\
\phi_{i}(r), \psi_{i}(r) \rightarrow 0 \text { as } r \rightarrow+\infty
\end{array}\right.
$$

is one-dimensional.

Suppose that $\left(\phi_{i}, \psi_{i}\right)$ solve (3.9). We must have

$$
\phi_{i}(0)=\psi_{i}(0)=0 .
$$

Similar to the proof of Claim 1, we see that either $\phi_{i}=\psi_{i} \equiv 0$ or $\phi_{i}<0, \psi_{i}<0$ for all $r$ or $\phi_{i}>0, \psi_{i}>0$ for all $r$. Since $\left(u^{\prime}, v^{\prime}\right)$ satisfies (3.9), by linearity of (3.9), 
$\left(\phi_{i}, \psi_{i}\right)-c\left(u^{\prime}, v^{\prime}\right)$ also satisfies (3.9). Let $c_{0}=\frac{\phi_{i}(1)}{u^{\prime}(1)}$. Then $\phi_{i}(1)-c u^{\prime}(1)=0$. Thus $\left(\phi_{i}, \psi_{i}\right)=c_{0}\left(u^{\prime}, v^{\prime}\right)$. This proves Claim 2 .

Theorem 2.1 follows from Claim 1 and Claim 2.

Remark. There is an alternative proof of this result for $\beta>0$ which may be useful for generalizations. One can easily show by the variational characterization of eigenvalues that the result will follow if we prove that the smallest eigenvalue of the system (3.9) is zero (by using the variational structure). Since we can easily show that the least eigenvalue of (3.9) has a nonnegative eigenfunction (by showing that replacing a test function $(h, k)$ by $(|h|,|k|)$ decreases the energy) and since we can use orthogonality to prove that there cannot be two distinct eigenvalues of the eigenvalue problem for (3.9) having nonnegative eigenfunctions, zero must be the least eigenvalue of the eigenvalue problem of (3.9) as claimed. (Note that $\left(-u^{\prime}(r),-v^{\prime}(r)\right)$ is a nonnegative eigenfunction of (3.9) corresponding to the eigenvalue zero.)

Completion of the Proof of Theorem 1.3. Theorem 1.3 follows directly from Theorem 2.1 and Theorem 3.1

\section{Approximate solutions and energy computations}

In this section we introduce some notation and present some preliminary analysis on approximate solutions.

From now on, we assume that $\beta \notin I \cup\left\{\beta_{1}, \ldots, \beta_{n}, \ldots.\right\}$ as in Theorem 1.3

Without loss of generality, we may assume that $0 \in \Omega$. By the following rescaling:

$$
x=\epsilon z, z \in \Omega_{\epsilon}:=\{\epsilon z \in \Omega\},
$$

equation (1.2) becomes

$$
\left\{\begin{array}{l}
\Delta u-\lambda_{1} u+\mu_{1} u^{3}+\beta u v^{2}=0 \text { in } \Omega_{\epsilon}, \\
\Delta v-\lambda_{2} v+\mu_{2} v^{3}+\beta u^{2} v=0 \text { in } \Omega_{\epsilon}, \\
u, v>0 \text { in } \Omega_{\epsilon}, \quad u=v=0 \text { on } \partial \Omega_{\epsilon} .
\end{array}\right.
$$

For $u, v \in H^{2}\left(\Omega_{\epsilon}\right) \cap H_{0}^{1}\left(\Omega_{\epsilon}\right)$, we put

$$
S_{\epsilon}\left(\begin{array}{l}
u \\
v
\end{array}\right)=\left(\begin{array}{c}
S_{1}(u, v) \\
S_{2}(u, v)
\end{array}\right)
$$

where $S_{1}(u, v)=\Delta u-\lambda_{1} u+\mu_{1} u^{3}+\beta u v^{2}$ and $S_{2}(u, v)=\Delta v-\lambda_{2} v+\mu_{2} v^{3}+\beta u^{2} v$.

Then solving system (4.2) is equivalent to

$$
S_{\epsilon}\left(\begin{array}{l}
u \\
v
\end{array}\right)=0, \quad u \in H^{2}\left(\Omega_{\epsilon}\right) \cap H_{0}^{1}\left(\Omega_{\epsilon}\right), \quad v \in H^{2}\left(\Omega_{\epsilon}\right) \cap H_{0}^{1}\left(\Omega_{\epsilon}\right) .
$$

Associated with problem (4.2) is the following energy functional:

$$
J_{\epsilon}[u]=\frac{1}{2} \int_{\Omega_{\epsilon}}\left(|\nabla u|^{2}+\lambda_{1} u^{2}+|\nabla v|^{2}+\lambda_{2} v^{2}\right)-\int_{\Omega_{\epsilon}}\left[\frac{1}{4} u^{4}+\frac{\beta}{2} u^{2} v^{2}+\frac{1}{4} v^{4}\right], u, v \in H_{0}^{1}\left(\Omega_{\epsilon}\right) .
$$

We define an inner product

$$
\left\langle\left(u_{1}, u_{2}\right),\left(v_{1}, v_{2}\right)\right\rangle_{\epsilon}=\int_{\Omega_{\epsilon}}\left(u_{1} v_{1}+u_{2} v_{2}\right), \text { for } u_{i}, v_{i} \in L^{2}\left(\Omega_{\epsilon}\right), i=1,2,
$$


and a configuration space

$$
\Lambda:=\{P \in \Omega \mid d(P, \partial \Omega)>\delta\}
$$

where $\delta$ is small.

Let $\left(U_{1}, U_{2}\right)$ be the solution of (1.4) which satisfies Theorem 1.3. For each $i=1,2$, by [35], $U_{i}$ is radially symmetric; $U_{i}(y)=U_{i}(|y|)$ and is strictly decreasing; $U_{i}^{\prime}(r)<0$ for $r>0, r=|y|$. Moreover, we have the following asymptotic behavior of $U_{i}$ :

$$
U_{i}(r)=A_{i} r^{-\frac{N-1}{2}} e^{-\sqrt{\lambda_{i}} r}\left(1+O\left(\frac{1}{r}\right)\right), \quad U_{i}^{\prime}(r)=-A_{i} \sqrt{\lambda_{i}} r^{-\frac{N-1}{2}} e^{-r}\left(1+O\left(\frac{1}{r}\right)\right),
$$

for $r$ large, where $A_{i}>0$ is a constant.

For $Q \in \Omega$, we define $U_{1, \epsilon, Q}$ to be the unique solution of

$$
\Delta u-\lambda_{1} u+\left[\mu_{1} U_{1}^{3}+\beta U_{1} U_{2}^{2}\right]\left(\cdot-\frac{Q}{\epsilon}\right)=0 \text { in } \Omega_{\epsilon}, \quad u=0 \text { on } \partial \Omega_{\epsilon} .
$$

Similarly, we set $U_{2, \epsilon, Q}$ to be the unique solution of

$$
\Delta v-\lambda_{2} v+\left[\mu_{2} U_{2}^{3}+\beta U_{2} U_{1}^{2}\right]\left(\cdot-\frac{Q}{\epsilon}\right)=0 \text { in } \Omega_{\epsilon}, \quad v=0 \text { on } \partial \Omega_{\epsilon} .
$$

Without loss of generality, we may assume that

$$
\lambda_{1} \leq \lambda_{2} .
$$

We first analyze $U_{1, \epsilon, Q}$. To this end, set

$$
\varphi_{1, \epsilon, Q}(x)=U_{1}\left(\frac{|x-Q|}{\epsilon}\right)-U_{1, \epsilon, Q}\left(\frac{x}{\epsilon}\right) .
$$

Then $\varphi_{1, \epsilon, Q}$ satisfies

$$
\Delta v-\lambda_{2} v=0 \text { in } \Omega_{\epsilon}, \quad v=U_{1}\left(\frac{|x-Q|}{\epsilon}\right) \text { on } \partial \Omega_{\epsilon} .
$$

Using (4.7) and modifying the proof in Section 4 of [32], we obtain the following lemma

Lemma 4.1.

(2) Let $V_{\varepsilon, i}(y)=\frac{\varphi_{i, \varepsilon, Q}(Q+\varepsilon y)}{\varphi_{i, \varepsilon, Q}(Q)}$. Then as $\varepsilon \rightarrow 0$ (up to a subsequence), $V_{\varepsilon, i}(y) \rightarrow$ $V_{i}(y)$, where $V_{i}(y)$ is a solution of

$$
\left\{\begin{array}{l}
\Delta V_{i}-\lambda_{i} V_{i}=0 \text { in } \mathbb{R}^{N} \\
V_{i}(0)=1, V_{i}>0 \text { in } \mathbb{R}^{N}
\end{array}\right.
$$

$$
\sup _{y \in \Omega_{\varepsilon, P}}\left|e^{-\sqrt{\lambda_{i}}(1+\sigma)|y|} V_{\varepsilon, i}(y)\right| \leq C \text { for any } 0<\sigma<1
$$

Using (4.1), we obtain the following error and energy estimates. 
Lemma 4.2. Assume that $Q \in \Lambda$. Then we have

$$
\left|S_{1}\left(U_{1, \epsilon, Q}, U_{2, \epsilon, Q}\right)\right|+\left|S_{2}\left(U_{1, \epsilon, Q}, U_{2, \epsilon, Q}\right)\right| \leq C\left(\varphi_{1, \epsilon, Q}+\varphi_{2, \epsilon, Q}\right)
$$

where $I_{0}=\frac{1}{4}\left[\mu_{1} U_{1}^{4}+2 \beta U_{1}^{2} U_{2}^{2}+\mu_{2} U_{2}^{4}\right], a_{1}=\frac{1}{2} \int_{\mathbb{R}^{N}}\left(\mu_{1} U_{1}^{3}+\beta U_{1} U_{2}^{2}\right) e^{\sqrt{\lambda_{1}} y_{1}}, a_{2}=$ $\frac{1}{2} \int_{\mathbb{R}^{N}}\left(\mu_{2} U_{2}^{3}+\beta U_{2} U_{1}^{2}\right) e^{\sqrt{\lambda_{2}} y_{1}}$.

Proof. (1) Since $\lambda_{1} \leq \lambda_{2}$, we estimate $S_{2}\left(U_{1, \epsilon, Q}, U_{2, \epsilon, Q}\right)$ first:

$$
\begin{aligned}
S_{2}\left(U_{1, \epsilon, Q}, U_{2, \epsilon, Q}\right) & =\Delta U_{2, \epsilon, Q}-\lambda_{2} U_{2, \epsilon, Q}+\mu_{2} U_{2, \epsilon, Q}^{3}+\beta U_{1, \epsilon, Q}^{2} U_{2, \epsilon, Q} \\
& =\mu_{2}\left(U_{2, \epsilon, Q}^{3}-U_{2}^{3}\right)+\beta\left(U_{1, \epsilon, Q}^{2} U_{2, \epsilon, Q}^{2}-U_{1} U_{2}^{2}\right) .
\end{aligned}
$$

The first term in (4.17) can be estimated easily:

$$
\left|U_{2, \epsilon Q}^{3}-U_{2}^{3}\right| \leq C U_{2}^{2} \varphi_{2, \epsilon, Q} \leq C \varphi_{2, \epsilon, Q}(Q)
$$

by Lemma 4.1 (3). Similarly, we can estimate other terms in (4.17) except the term $U_{1}^{2} \varphi_{2, \epsilon, Q}$. If $\lambda_{1}=\lambda_{2}$, we have $\varphi_{2, \epsilon, Q} \leq \varphi_{1, \epsilon, Q}$. If $\lambda<\lambda_{2}$, we also have $\varphi_{2, \epsilon, Q} \leq \varphi_{1, \epsilon, Q}$ by the comparison principle. In any case, we obtain

$$
U_{1}^{2} \varphi_{2, \epsilon, Q} \leq C U_{1}^{2} \varphi_{1, \epsilon, Q} \leq C \varphi_{1, \epsilon, Q}(Q) .
$$

Similarly, we can estimate $S_{1}\left(U_{1, \epsilon, Q}, U_{2, \epsilon, Q}\right)$.

(2) Using the definitions of $U_{i, \epsilon, Q}$, we obtain

$$
\begin{aligned}
J_{\epsilon}\left[U_{1, \epsilon, Q}, U_{2, \epsilon, Q}\right]= & \frac{1}{2} \int_{\Omega_{\epsilon}}\left(\mu_{1} U_{1}^{3}+\beta U_{1} U_{2}^{2}\right)\left(U_{1}-\varphi_{1, \epsilon, Q}\right) \\
& +\frac{1}{2} \int_{\Omega_{\epsilon}}\left(\mu_{2} U_{2}^{3}+\beta U_{2} U_{1}^{2}\right)\left(U_{2}-\varphi_{2, \epsilon, Q}\right) \\
& -\frac{1}{4} \int_{\Omega_{\epsilon}}\left(\mu_{1} U_{1, \epsilon, Q}^{4}+2 \beta U_{1, \epsilon, Q}^{2} U_{2, \epsilon, Q}^{2}+\mu_{2} U_{2, \epsilon, Q}^{4}\right) \\
= & \frac{1}{4} \int_{\mathbb{R}^{N}}\left[\mu_{1} U_{1}^{4}+2 \beta U_{1}^{2} U_{2}^{2}+\mu_{2} U_{2}^{4}\right] \\
& -\frac{1}{2} \int_{\Omega_{\epsilon}}\left[\mu_{1} U_{1}^{3}+\beta U_{1} U_{2}^{2}\right] \varphi_{1, \epsilon, Q}-\frac{1}{2} \int_{\Omega_{\epsilon}}\left[\mu_{2} U_{2}^{3}+\beta U_{1}^{2} U_{1}\right] \varphi_{2, \epsilon, Q} \\
& +O\left(e^{-(2 \sqrt{\lambda}+\delta) \frac{d(Q, \partial \Omega)}{\epsilon}}\right) .
\end{aligned}
$$

By Lemma 4.1 (2), we have

$$
\frac{1}{2} \int_{\Omega_{\epsilon}}\left[\mu_{1} U_{1}^{3}+\beta U_{1} U_{2}^{2}\right] \varphi_{1, \epsilon, Q}=\varphi_{1, \epsilon, Q}(Q) \frac{1}{2} \int_{\Omega_{\epsilon}}\left[\mu_{1} U_{1}^{3}+\beta U_{1} U_{2}^{2}\right] V_{\epsilon, 1},
$$

where

$$
\int_{\Omega_{\epsilon}}\left[\mu_{1} U_{1}^{3}+\beta U_{1} U_{2}^{2}\right] V_{\epsilon, i} \rightarrow \int_{\mathbb{R}^{N}}\left[\mu_{1} U_{1}^{3}+\beta U_{1} U_{2}^{2}\right] V_{1}=\int_{\mathbb{R}^{N}}\left[\mu_{1} U_{1}^{3}+\beta U_{1} U_{2}^{2}\right] e^{\sqrt{\lambda_{1}} y_{1}}
$$

by Lemma 4.7 of [32. Similarly, we have

$$
\int_{\Omega_{\epsilon}}\left[\mu_{2} U_{2}^{3}+\beta U_{1}^{2} U_{1}\right] \varphi_{2, \epsilon, Q}=\varphi_{2, \epsilon, Q}\left(\int_{\mathbb{R}^{N}}\left[\mu_{2} U_{2}^{3}+\beta U_{2} U_{1}^{2}\right] e^{\sqrt{\lambda_{2}} y_{1}}+o(1)\right) .
$$

This proves (4.16). 


\section{Lochlized ENERgy Method AND PRoOf of Theorem 1.2}

In this section we develop the linear theory which allows us to perform the finitedimensional reduction procedure. Here we need the nondegeneracy result Theorem 1.3 .

Fix $Q \in \Lambda$. We define the following functions:

$$
\begin{gathered}
Z_{i, j}=\frac{\partial U_{i}}{\partial z_{j}} \chi\left(\frac{4 \epsilon}{\delta}\left|z-\frac{Q}{\epsilon}\right|\right), \quad i=1,2, j=1, \ldots, N, \\
Z_{j}=\left(\begin{array}{c}
Z_{1, j} \\
Z_{2, j}
\end{array}\right), \quad j=1,2, \ldots, N,
\end{gathered}
$$

where $\chi(t)$ is a smooth cut-off function such that $\chi(t)=1$ for $|t|<1$ and $\chi(t)=0$ for $|t|>2$.

We first consider the following linear problem: Let

$$
L_{\epsilon}\left(\begin{array}{c}
\phi \\
\psi
\end{array}\right):=\left(\begin{array}{c}
\Delta \phi-\lambda_{1} \phi+\left(3 \mu_{1} U_{1, \epsilon, Q}^{2}+U_{2, \epsilon, Q}^{2}\right) \phi+2 \beta U_{1, \epsilon, Q} U_{2, \epsilon, Q} \psi \\
\Delta \psi-\lambda_{1} \psi+\left(3 \mu_{2} U_{2, \epsilon, Q}^{3}+U_{1, \epsilon, Q}^{2}\right) \psi+2 \beta U_{1, \epsilon, Q} U_{2, \epsilon, Q} \phi
\end{array}\right) .
$$

Given $h_{1}, h_{2} \in L^{2}\left(\Omega_{\epsilon}\right)$, find a function $\left(\begin{array}{c}\phi \\ \psi\end{array}\right)$ satisfying

$$
\left\{\begin{array}{l}
L_{\epsilon}\left(\begin{array}{c}
\phi \\
\psi
\end{array}\right)=\left(\begin{array}{c}
h_{1} \\
h_{2}
\end{array}\right)+\sum_{j=1}^{N} c_{j} Z_{j}, \\
\left\langle\left(\begin{array}{c}
\phi \\
\psi
\end{array}\right), Z_{j}\right\rangle_{\epsilon}=0, j=1, \ldots, N, \quad \text { and } \phi=\psi=0 \text { on } \partial \Omega_{\epsilon},
\end{array}\right.
$$

for some constants $c_{l}, l=1, \ldots, N$. To this purpose, we define two norms

$$
\|\phi\|_{*}=\|\phi\|_{W^{2, q}\left(\Omega_{\epsilon}\right)}, \quad\|f\|_{* *}=\|f\|_{L^{q}\left(\Omega_{\epsilon}\right)},
$$

where $q>\frac{N}{2}$ is a fixed number.

We have the following result:

Proposition 5.1. Let $\phi$ satisfy (5.3). Then for $\epsilon$ sufficiently small and $\mathbf{Q} \in \Lambda$, we have

$$
\|\phi\|_{*}+\|\psi\|_{*} \leq C\left(\left\|h_{1}\right\|_{* *}+\left\|h_{2}\right\|_{* *}\right)
$$

where $C$ is a positive constant independent of $\epsilon$ and $Q \in \Lambda$.

Proof. We follow the proof of Proposition 3.1 of [24, p. 264]. Arguing by contradiction, assume that there a sequence $\left(\phi_{n}, \psi_{n}, h_{1 n}, h_{2 n}\right)$ satisfying (5.3) such that

$$
\left\|\phi_{n}\right\|_{*}+\left\|\psi_{n}\right\|_{*}=1 ; \quad\left\|h_{1 n}\right\|_{* *}+\left\|h_{2 n}\right\|=o(1) .
$$

To avoid clumsy notations, we omit the dependence on $n$.

Multiplying (5.3) by $Z_{j}$, we obtain

$$
\sum_{i=1}^{N} c_{i}\left\langle Z_{i}, Z_{j}\right\rangle_{\epsilon}=-\left\langle\left(\begin{array}{c}
h_{1} \\
h_{2}
\end{array}\right), Z_{j}\right\rangle_{\epsilon}+\left\langle L_{\epsilon}\left(\begin{array}{c}
\phi \\
\psi
\end{array}\right), Z_{j}\right\rangle, j=1, \ldots, N
$$

Since $U_{i, \epsilon, Q}=U_{i}+O\left(e^{-\frac{\delta}{\epsilon}}\right)$ and $\left\langle Z_{i}, Z_{j}\right\rangle_{\epsilon}=\left\langle Z_{1}, Z_{1}\right\rangle_{\epsilon} \delta_{i j}$, by integration by parts, we obtain

$$
c_{j}=o(1), j=1, \ldots, N
$$


Thus as $n \rightarrow+\infty$, we obtain $\left(\phi_{n}, \psi_{n}\right) \rightarrow\left(\phi_{0}, \psi_{0}\right)$ in $C_{l o c}^{0}$, where $\left(\phi_{0}, \psi_{0}\right)$ is a solution of

$$
\left\{\begin{array}{l}
\Delta \phi_{0}-\lambda_{1} \phi_{0}+\left(3 \mu_{1} U_{1}^{2}+U_{2}^{2}\right) \phi_{0}+2 \beta U_{1} U_{2} \psi_{0}=0 \\
\Delta \psi_{0}-\lambda_{2} \psi_{0}+\left(3 \mu_{2} U_{2}^{3}+U_{1}^{2}\right) \psi_{0}+2 \beta U_{1} U_{2} \phi_{0}=0 \\
\left|\phi_{0}\right|+\left|\psi_{0}\right| \leq 1
\end{array}\right.
$$

By Theorem 1.3, $\phi_{0} \equiv 0, \psi_{0} \equiv 0$. This implies by the Lebesgue Dominated Convergence Theorem that

$$
\left\|\left(3 \mu_{1} U_{1, \epsilon, Q}^{2}+U_{2, \epsilon, Q}^{2}\right) \phi_{\epsilon}+2 \beta U_{1, \epsilon, Q} U_{2, \epsilon, Q} \psi_{\epsilon}\right\|_{L^{q}\left(\Omega_{\epsilon}\right)}=o(1) .
$$

By elliptic regularity, $\left\|\phi_{\epsilon}\right\|_{W^{2, q}\left(\Omega_{\epsilon}\right)}=o(1)$. Similarly, we obtain $\left\|\psi_{\epsilon}\right\|_{L^{q}\left(\Omega_{\epsilon}\right)}=o(1)$. A contradiction.

By the Fredholm Alternative (see the proof of Proposition 3.2 of [24, p. 267]), we also obtain

Proposition 5.2. There exists $\epsilon_{0}>0$ such that for any $0<\epsilon<\epsilon_{0}$ the following property holds true. Given $h_{1}, h_{2} \in W^{2, q}\left(\Omega_{\epsilon}\right)$, there exists a unique pair $(\phi, \psi, \mathbf{c})=$ $\left(\phi,\left\{c_{j}\right\}_{j=1, \ldots, N}\right)$ such that

$$
\begin{gathered}
L_{\epsilon}\left(\begin{array}{c}
\phi \\
\psi
\end{array}\right)=\left(\begin{array}{c}
h_{1} \\
h_{2}
\end{array}\right)+\sum_{j} c_{j} Z_{j}, \\
\left\langle\left(\begin{array}{c}
\phi \\
\psi
\end{array}\right), Z_{j}\right\rangle_{\epsilon}=0, j=1, \ldots, N, \phi=\psi=0 \quad \text { on } \partial \Omega_{\epsilon} .
\end{gathered}
$$

Moreover, we have

$$
\|\phi\|_{*}+\|\psi\|_{*} \leq C\left\|h_{1}\right\|_{* *}+\left\|h_{2}\right\|_{* *}
$$

for some positive constant $C$.

Finally, we solve a nonlinear problem: For $\epsilon$ small and for $Q \in \Lambda$, we are going to find a function $\left(\begin{array}{c}\phi_{1, \epsilon, Q} \\ \phi_{2, \epsilon, Q}\end{array}\right)$ such that for some constants $c_{j}, j=1, \ldots, N$, the following equation holds true:

$$
\left\{\begin{array}{l}
S_{\epsilon}\left(U_{1, \epsilon, Q}+\phi_{1, \epsilon, Q}, U_{2, \epsilon, Q}+\phi_{2, \epsilon, Q}\right)=\sum_{l=1}^{N} c_{l} Z_{l} \text { in } \Omega_{\epsilon}, \\
\left\langle\left(\begin{array}{c}
\phi_{1, \epsilon, Q} \\
\phi_{2, \epsilon, Q}
\end{array}\right), Z_{j}\right\rangle_{\epsilon}=0, j=1, \ldots, N, \quad \phi=\psi=0 \text { on } \partial \Omega_{\epsilon} .
\end{array}\right.
$$

Using Lemma 4.2 and the Contraction Mapping Principle, similar to the proof of Proposition 4.2 of [24, p. 268], we obtain

Proposition 5.3. For $Q \in \Lambda$ and $\epsilon$ sufficiently small, there exists a unique $\left(\begin{array}{c}\phi_{1, \epsilon, Q} \\ \phi_{2, \epsilon, Q}\end{array}\right)$ such that (5.13) holds. Moreover, $Q \mapsto\left(\begin{array}{c}\phi_{1, \epsilon, Q} \\ \phi_{2, \epsilon, Q}\end{array}\right)$ is of class $C^{1}$ as a map into $W^{2, q}\left(\Omega_{\epsilon}\right)$, and we have

$$
\sum_{i=1}^{2}\left\|\phi_{i, \epsilon, \mathbf{Q}}\right\|_{*} \leq C\left(\varphi_{1, \epsilon, Q}(Q)+\varphi_{2, \epsilon, Q}(Q)\right)
$$

for some constant $C>0$. 


\section{Proof of Theorem 1.2}

In this section, we prove Theorem 1.2 .

We first present a reduction lemma. Fix $\mathbf{Q} \in \Lambda$. Let $\left(\phi_{1, \epsilon, Q}, \phi_{2, \epsilon, Q}\right)$ be the solution given by Proposition 5.3. We define a new functional

$$
\mathcal{M}_{\epsilon}(Q)=J_{\epsilon}\left[U_{1, \epsilon, Q}+\phi_{1, \epsilon, Q}, U_{2, \epsilon, Q}+\phi_{2, \epsilon, Q}\right]: \Lambda \rightarrow R .
$$

Then we have (similar to the proof of Theorem 1.1 of [24, p. 271])

Lemma 6.1. If $Q_{\epsilon}$ is a critical point of $\mathcal{M}_{\epsilon}(Q)$ in $\Lambda$, then $\left(U_{1, \epsilon, Q_{\epsilon}}+\phi_{1, \epsilon, Q_{\epsilon}}, U_{2, \epsilon, Q_{\epsilon}}+\right.$ $\left.\phi_{2, \epsilon, Q_{\epsilon}}\right)$ is a critical point of $J_{\epsilon}$ and hence a solution to (4.2).

Therefore, the proof of Theorem 1.2 is finished after we have the following proposition:

Proposition 6.2. For $\epsilon$ small, the minimization problem

$$
\min \left\{\mathcal{M}_{\epsilon}(Q): Q \in \Lambda\right\}
$$

has a solution $Q^{\epsilon} \in \Lambda^{\circ}$ the interior of $\Lambda$. Furthermore,

$$
d\left(Q_{\epsilon}, \partial \Omega\right) \rightarrow \max _{P \in \Omega} d(P, \partial \Omega) .
$$

Proof. Since $J_{\epsilon}\left[U_{1, \epsilon, Q}+\phi_{1, \epsilon, Q}, U_{2, \epsilon, Q}+\phi_{2, \epsilon, Q}\right]$ is continuous in $Q$, the minimization problem has a solution. Let $\mathcal{M}_{\epsilon}\left(Q_{\epsilon}\right)$ be the minimum where $Q_{\epsilon} \in \Lambda$.

We claim that $Q_{\epsilon}$ must stay in the interior of $\Lambda$.

We first obtain an asymptotic formula for $\mathcal{M}_{\epsilon}(Q)$. In fact for any $Q \in \Lambda$, we have

$$
\begin{gathered}
\mathcal{M}_{\epsilon}(Q)=J_{\epsilon}\left[U_{1, \epsilon, Q}, U_{2, \epsilon, Q}\right] \\
+\int_{\Omega_{\epsilon}}\left(-S_{1}\left[U_{1, \epsilon, Q}, U_{2, \epsilon, Q}\right] \phi_{1, \epsilon, Q}-S_{2}\left[U_{1, \epsilon, Q}, U_{2, \epsilon, Q}\right] \phi_{2, \epsilon, Q}\right)+O\left(\sum_{i=1}^{2}\left\|\phi_{i, \epsilon, \mathbf{Q}}\right\|_{*}^{2}\right) \\
=I_{0}+\sum_{i=1}^{2}\left(a_{i}+o(1)\right) \varphi_{i, \epsilon, Q}(Q)
\end{gathered}
$$

by Lemma 4.2 and Proposition 5.3 .

First, by choosing $Q_{0}$ such that $d\left(Q_{0}, \partial \Omega\right)=\max _{P \in \Omega} d(P, \partial \Omega)$, we obtain a lower bound for $\mathcal{M}_{\epsilon}$ :

$$
\mathcal{M}_{\epsilon}\left(Q_{\epsilon}\right) \leq I_{0}+\sum_{i=1}^{2}\left(a_{i}+o(1)\right) \varphi_{i, \epsilon, Q_{0}}\left(Q_{0}\right),
$$

which, by (6.3) and Lemma 4.1, gives

$$
\lim _{\epsilon \rightarrow 0} d\left(Q_{\epsilon}, \partial \Omega\right) \geq d\left(Q_{0}, \partial \Omega\right) .
$$

Proposition 6.2 is thus proved.

\section{ACKNOWLEDGMENTS}

The research of the first author was partially supported by the ARC, while the second author was partially supported by an Earmarked Grant from RGC of Hong Kong. We thank the referees for carefully reading the manuscript and for many suggestions. 


\section{REFERENCES}

[1] A. Ambrosetti and E. Colorado, Bound and ground states of coupled nonlinear Schrodinger equations, C. R. Math. Acad. Sci. Paris 342 (2006), 453-458. MR2214594 (2006j:35057)

[2] N. Akhmediev and A. Ankiewicz, Partially coherent solitons on a finite background, Phys. Rev. Lett. 82 (1998), 2661, 1-4.

[3] T. Bartsch, Z.-Q. Wang and J. Wei, Bound states for a coupled Schrödinger system, JFEPT, to appear.

[4] P. Bates, E.N. Dancer and J. Shi, Multi-spike stationary solutions of the Cahn-Hilliard equation in higher-dimension and instability, Adv. Differential Equations 4 (1999), 1-69. MR.1667283 (99k:35097)

[5] P. Bates and J. Shi, Existence and instability of spike layer solutions to singular perturbation problems, J. Funct. Anal. 196 (2002), 211-264. MR1943093 (2003k:35045)

[6] S. Chang, C.S. Lin, T.C. Lin and W. Lin. Segregated nodal domains of two-dimensional multispecies Bose-Einstein condensates. Phys. D 196(3-4) (2004), 341-361. MR2090357 (2005g:82074)

[7] D.N. Christodoulides, T.H. Coskun, M. Mitchell and M. Segev, Theory of incoherent selffocusing in biased photorefractive media, Phys. Rev. Lett. 78(1-4) (1997), 646.

[8] M. Crandall and P. Rabinowitz, Bifurcation from simple eigenvalues, J. Functional Analysis 8 (1971), 321-340. MR0288640 (44:5836)

[9] M. Crandall and P. Rabinowitz, Bifurcation, perturbation of simple eigenvalues and linearized stability, Arch. Rational Mech. Anal. 52 (1973), 161-180. MR0341212 (49:5962)

[10] E.N. Dancer, Boundary-value problems for ordinary differential equations on infinite intervals, Proc. London Math. Soc. 30 (1975), 76-94. MR0379963 (52:867)

[11] E.N. Dancer, Real analyticity and non-degeneracy, Math. Ann. 325 (2003), no. 2, 369-392. MR1962054 (2004h:35067)

[12] E.N. Dancer, Global structure of the solutions of nonlinear real analytic eigenvalue problems, Proc. London Math. Soc. 27 (1973), 747-765. MR0375019(51:11215)

[13] E.N. Dancer and S. Yan, Multipeak solutions for a singular perturbed Neumann problem, Pacific J. Math. 189 (1999), 241-262. MR.1696122 (2000d:35010)

[14] E.N. Dancer and S. Yan, Interior and boundary peak solutions for a mixed boundary value problem, Indiana Univ. Math. J. 48 (1999), 1177-1212. MR1757072(2001f:35146)

[15] M. del Pino, P. Felmer, M. Musso, Two-bubble solutions in the super-critical Bahri-Coron's problem, Cal. Var. PDE 16 (2003), no.2, 113-145. MR1956850 (2004a:35079)

[16] C. Gui and J. Wei, Multiple interior spike solutions for some singular perturbed Neumann problems, J. Diff. Eqns. 158 (1999), 1-27. MR1721719 (2000g:35035)

[17] C. Gui and J. Wei, On multiple mixed interior and boundary peak solutions for some singularly perturbed Neumann problems, Can. J. Math. 52 (2000), 522-538. MR 1758231 (2001b:35023)

[18] C. Gui, J. Wei and M. Winter, Multiple boundary peak solutions for some singularly perturbed Neumann problems, Ann. Inst. H. Poincaré Anal. Non Linéaire 17 (2000), 249-289. MR.1743431 (2001a:35018)

[19] B.D. Esry, C.H. Greene, J.P. Burke, Jr., J.L. Bohn, Hartree-Fock theory for double condensates, Phys. Rev. Lett. 78 (1997), 3594-3597.

[20] B. Gidas and J. Spruck, Global and local behavior of positive solutions of nonlinear elliptic equations, Comm. Pure Appl. Math. 35 (1981), 525-598. MR615628 (83f:35045)

[21] F.T. Hioe, Solitary Waves for $N$ Coupled Nonlinear Schrödinger Equations, Phys. Rev. Lett. 82 (1999), 1152-1155.

[22] F.T. Hioe, T.S. Salter, Special set and solutions of coupled nonlinear Schrödinger equations, J. Phys. A: Math. Gen. 35 (2002), 8913-8928. MR1946865(2003k:35230)

[23] T. Kanna and M. Lakshmanan, Exact soliton solutions, shape changing collisions, and partially coherent solitons in coupled nonlinear Schrödinger equations, Phys. Rev. Lett. 86 (1-4) (2001), 5043.

[24] F.H. Lin, W.M. Ni and J. Wei, On the number of interior peak solutions for a singularly perturbed Neumann problem, Comm. Pure Appl. Math. 60 (2007), no. 2, 252-281. MR 2275329

[25] T.-C. Lin and J.-C. Wei, Ground state of $N$ coupled Nonlinear Schrödinger Equations in $R^{n}, n \leq 3$, Communications in Mathematical Physics 255(3) (2005), 629-653. MR2135447 (2006g:35044) 
[26] T.-C. Lin and J. Wei, Spikes in two coupled nonlinear Schrödinger equations, Ann. I. H. Poincaré Analyse. Non. 22(4) (2005), 403-439. MR2145720(2006a:35065)

[27] T.-C. Lin and J. Wei, Solitary and self-similar solutions of two-component system of nonlinear Schrödinger equations, Physica D: Nonlinear Phenomena. 220 (2006), no. 2, 99-115. MR2253405 (2007d:35241)

[28] L.A. Maia, E. Montefusco and B. Pellacci, Positive solutions for a weakly coupled nonlinear Schrödinger system, J. Diff. Eqns. 229 (2006), no. 2, 743-767. MR2263573 (2007h:35070)

[29] M. Mitchell, Z. Chen, M. Shih, and M. Segev, Self-trapping of partially spatially incoherent light, Phys. Rev. Lett. 77 (1996), 490-493.

[30] M. Mitchell and M. Segev, Self-trapping of incoherent white light, Nature 387 (1997), 880-882.

[31] W.M. Ni and I. Takagi, Locating the peaks of least energy solutions to a semilinear Neumann problem, Duke Math. J. 70 (1993), 247-281. MR1219814 (94h:35072)

[32] W.-M. Ni and J. Wei, On the location and profile of spike-layer solutions to singularly perturbed semilinear Dirichlet problems, Comm. Pure Appl. Math. 48 (1995), 731-768. MR:1342381 (96g:35077)

[33] B. Sirakov, Least energy solitary waves for a system of nonlinear Schrodinger equations, Comm. Math. Phys. 271 (2007), 199-221. MR2283958 (2007k:35477)

[34] E. Timmermans, Phase separation of Bose-Einstein condensates, Phys. Rev. Lett. 81 (1998), $5718-5721$

[35] W. C. Troy, Symmetry properties in systems of semilinear elliptic equations, J. Diff. Eqn. 42(3) (1981), 400-413. MR639230 (83b:35051)

[36] J. Wei, On the construction of single-peaked solutions to a singularly perturbed semilinear Dirichlet problem, J. Differential Equations 129 (1996), 315-333. MR.1404386 (97f:35015)

School of Mathematics and Statistics, University of Sydney, Sydney, Australia

E-mail address: normd@maths.usyd.edu.au

Department of Mathematics, The Chinese University of Hong Kong, Shatin, Hong KONG

E-mail address: wei@math.cuhk.edu.hk 